\title{
Deriving a Mechanistic Model for Potential Energy Surface of Coordination Compounds of Nontransition Elements
}

\author{
A. L. TCHOUGRÉEFF ${ }^{1,2}$ \\ ${ }^{1}$ Division of Electrochemistry, Department of Chemistry, Moscow State University, Moscow, Russia \\ ${ }^{2}$ Karpov Institute of Physical Chemistry, Moscow, Russia
}

Received 5 February 2007; accepted 12 April 2007

Published online 1 June 2007 in Wiley InterScience (www.interscience.wiley.com).

DOI 10.1002/qua.21421

\begin{abstract}
Molecules of coordination compounds (those formed by a central atom-largely by a transition or non-transition metal ion, but also by non-transition elements like sulfur, phosphorus, etc., and by atoms or groups surrounding it-the ligands) for decades represent a significant problem for any "classical" description given in terms of empirical force fields of molecular mechanics (MM) due to diversity of coordination polyhedra resulting in the numerosity of the parameters necessary to describe the objects of interest within such a setting. This situation is further toughened by the specific collection of effects known as mutual influence of ligands, of which the trans-effect in transition metal complexes is the most known. A feature particularly complicating understanding the ligand influence is its qualitative dependence on the nature of the central atom. The real source of these problems is of course the specificity of the electronic structure of the coordination compounds. If compared with usual organic molecules for which the MM in its classical form is rather successful the major distinction characteristic for coordination compounds is the absence of fairly distinguishable two-center two-electron bonds incident to its central atom (ion). Using a methodology called deductive molecular mechanics (DMM) we have recently shown that for "organic" molecules it is possible to sequentially derive the form of the MM force field departing from a simple but intuitively transparent model of electronic structure of a single chemical bond. Here we present an analogous derivation for the
\end{abstract}

Correspondence to: A. L. Tchougréeff; e-mail: andrei@cc.nifhi. ac.ru

Contract grant sponsor: RFBR.

Contract grant numbers: 04-03-32146, 04-03-32206, 05-0790067, 07-03-01128. 


\title{
TCHOUGRÉEFF
}

\begin{abstract}
force fields describing coordination compounds. It is based on the analysis of electronic structure of the closest ligand shell of coordination compounds and on that of its relation with the geometry changes induced by chemical substitution (ligand influence) performed recently by Levin and Dyachkov. By using the elements of the one-electron density matrix as an economical set of electronic structure variables for the closest ligand shell, the DMM model of coordination compounds is constructed. Next, by excluding the electronic structure variables the effective elastic elements describing the force fields concordant with the mutual ligands' influence in coordination compounds are constructed and their dependence on the state of the central ion is established. (C) 2007 Wiley Periodicals, Inc. Int J Quantum Chem 107: 2519-2538, 2007
\end{abstract}

Key words: deductive molecular mechanics; coordination compounds; models of potential energy surface

\section{Introduction}

C onstructing a mechanistic model for potential energy surfaces (PES) of coordination compounds, which would be consistent with the ligand influence effects in this class of molecules, for decades, remains actual for the respective area of computational chemistry [1]. Modern progress in quantum chemistry allows to make reliable predictions for the PES of polyatomic molecular systems including coordination compounds on the basis of direct calculations of their electronic structure. However, high-level methods, like ab initio and DFT, are computationally too demanding when dealing with coordination compounds and incidentally do not provide any theoretical insight into physical reasons controlling the observable (and hopefully numerically reproducible) behavior of these species. On the other hand, qualitative concepts, such as points-on-a-sphere (POS) [2] or metal-ligand size-match selectivity [3], playing important roles in theoretical coordination chemistry of both transition and non-transition metals, miss any reliable semi-quantitative structure-energy relation. Thus, effective numerical tools suitable for semi-empirical modeling of coordination compounds and reproducing the qualitative aspects of their stereochemistry (including ligand influence) are strongly in demand. Recent studies reviewed in [4] are devoted to analysis of chemical bonding in various coordination compounds. They are, however, mainly focused on truly covalent compounds with more or less well-distinguishable two-center bonds. By virtue of this the electronic structure of compounds considered in [4] differs on an intuitive level from the picture of unsaturable and undirected "coordination" bonds characteristic for com- plexes of metals with organic ligands with donor atoms [5]. Here we assume to consider namely this latter class of compounds from the point of view of possibility of constructing a mechanistic model of their PESs based on a sequential quantum model of electronic structure of their closest coordination spheres. Before plunging into this we explain what has to be expected from the theory to be constructed here.

It is known from the literature [6] that in coordination compounds due to mutual ligand influence effects the MM parameters involving the central atom (ion) are that numerous that any mechanistic description of them with an acceptable result makes the entire enterprise eventually senseless. There would be no chance to get any insight to this diversity from the scratch. Fortunately, A. A. Levin and P. N. Dyachkov being based in the I. B. Bersuker theory of vibronic interactions [7] yet in 1980s performed an exhaustive qualitative analysis of the interplay between chemical substitutions and deformations of the complex geometry, i.e. of the ligand influence in those of both transition and non-transition elements [8] for the most widespread coordination polyhedra: octahedron, tetrahedron, and planar square. By this they established basic relations between the characteristics of electronic structure of coordination compounds and their geometry. Analysis performed in [8] reduces to qualitative reasoning on the properties of the solutions of the MO LCAO method in the restricted basis of functions. Formally it is a special case of applying the M. J. S. Dewar's theory of perturbations of MOs [9] to the special class of the MOs residing in the closest ligand shell (CLS) of a coordination compound. Our purpose in the present paper is to develop such a formal representation to the results of [8], which could be recast into a mech- 
anistic view upon the PES of coordination compounds in terms of classically looking force fields derived from the quantum mechanical picture of molecular electronic structure. By this we theoretically derive an MM-like description covering coordination compounds and give an explanation and introduce systematization into diversity of their parameters.

\section{Ligand Influence From MM Perspective}

In the standard MM [10] setting the substitution effects upon molecular geometry (ligand influence) can be only awkwardly described. The PES in the MM setting is the sum

$$
E=E_{\mathrm{b}}+E_{\text {ang }}+E_{\text {tors }}+E_{\mathrm{nb}}
$$

of the bond stretching $\left(E_{\mathrm{b}}\right)$, valence angle bending $\left(E_{\text {ang }}\right)$, torsional $\left(E_{\text {tors }}\right)$, and nonbonding $\left(E_{\mathrm{nb}}\right)$ contributions (force fields), which are all explicit functions of nuclear coordinates. The nonbonding force field is mainly contributed by the Coulomb interactions of some effective charges and by the van der Waals forces:

$$
E_{\mathrm{nb}}=E_{\mathrm{Coul}}+E_{\mathrm{vdW}}
$$

The equilibrium nuclear coordinates $q_{\mathrm{eq}}$ coming from the MM approximation are defined by the equilibrium conditions, which can be conveniently reformulated as those of the evanescence of the forces acting on the atoms (in the MM context using this form of the equilibrium condition dating back to Sir Isaac Newton himself was suggested in Ref. [11]). In the standard MM setting the equilibrium interatomic distances are close to some ideal values $q_{0}$ (which are part of the parameters' sets describing respective force field) specific for the bonds of each considered type. In the vicinity of these ideal values the energy can be safely assumed (and it is frequently but not always done in the MM setting) to be quadratic in the nuclear coordinate shifts so that the actual values of the equilibrium interatomic distances (and of other geometry parameters) appear as a result of the response of the system to the presumably small forces coming from the nonbonding part of the molecular force field:

$$
\begin{aligned}
& q_{\mathrm{eq}}-q_{0}=D^{-1} f\left(q_{0}\right) \quad \text { where } \\
& \mathrm{f}\left(\mathrm{q}_{0}\right)=-\left.\nabla_{\mathrm{q}}\left(\mathrm{E}_{\text {tors }}+E_{\text {Coul }}+E_{\mathrm{vdW}}\right)\right|_{q=q_{0}}
\end{aligned}
$$

where by $\nabla_{q}$ we understand the first derivative (gradient) with respect to all the nuclear coordinates and $D^{-1}$ is the inverse of the dynamic matrix (that of the second derivatives of the energy) derived from the harmonic bonding contribution (bond stretching and valence angle bending) to the $\mathrm{MM}$ energy only. This approximation to the MM energy yields the diagonal dynamical matrix if the coordinates are taken as the bond lengths' and valence angles' shifts, so that $D^{-1}$ is also diagonal and can be easily calculated. Later we shall restrict ourselves to a model of octahedral complex where no quadruplet of atoms sequentially connected by bonds appears. That means that no proper torsion terms $E_{\text {tors }}$ actually appear in the expression for the energy Eq. (1). For that reason we do not consider them hereinafter. Under these conditions whatever coupling between the individual bond stretching and valence angle bending modes is only possible through the mediation of the nonbonding force fields unless it is introduced into the model explicitly. We are not going to insert these missing couplings "by hand" rather to derive them sequentially. Hence we first explore what the nonbonding force fields can give in this respect.

\section{COULOMB CONTRIBUTION TO LIGAND INFLUENCE}

Let us explore the possibility to ascribe the ligand influence to the nonbonding part of the classical force field starting from the leading Coulomb contribution to the energy. In the octahedral complex $\mathrm{ML}_{6}$ a single substitution $\mathrm{ML}_{6} \rightarrow \mathrm{ML}_{5} \mathrm{X}$ manifests itself in changing the ideal bond length of one of the $\mathrm{M}-\mathrm{L}$ bonds to that of the $\mathrm{M}-\mathrm{X}$ bond. Under the formulated conditions this does not affect directly the lengths of other $\mathrm{M}-\mathrm{L}$ bonds (the off-diagonal terms are absent), but changes the potentials of the force fields felt by other atoms L. Let us assume for the sake of definiteness that the replaced ligand occupies the apical position, i.e., takes a positive value of its $z$-coordinate in the coordinate frame centered at the $\mathrm{M}$ atom with the axes directed along the $\mathrm{M}-\mathrm{L}$ bonds. Next, we can set the ideal value of the $\mathrm{M}-\mathrm{X}$ bond $r_{\mathrm{MX}}$ to differ from that for the $\mathrm{M}-\mathrm{L}$ bonds $r_{\mathrm{ML}}$ by the quantity $\delta r_{\mathrm{XL}}$ : 


$$
r_{\mathrm{MX}}=r_{\mathrm{ML}}+\delta r_{\mathrm{XL}}
$$

and assume that the ideal position of the $X$ substituent does not deviate from the z-axis. We also assume that the effective charge of the $X$ substituent differs from that of the ligand $\mathrm{L}$ by an amount $\delta Q_{\mathrm{XL}}$. This value is not, however, absolutely independent since we assume that the effective charge of the central atom under the substitution changes by the same quantity $\delta Q_{\mathrm{XL}}$ in the opposite sense. Under these assumptions one can write the variation of the electrostatic potential caused by the substitution in the point $r$ of the three-dimensional space, which is enough distant from the substituent $X$. Retaining the terms of the first order with respect to either $\delta r_{\mathrm{XL}}$ or $\delta Q_{\mathrm{XL}}$ we get:

$$
\begin{aligned}
\delta \varphi_{\text {Coul }}(\mathbf{r})=\delta Q_{\mathrm{XL}}\left(\frac{1}{\left|\mathbf{r}-\mathbf{R}_{\mathrm{L}}\right|}\right. & \left.-\frac{1}{\left|\mathbf{r}-\mathbf{R}_{\mathrm{M}}\right|}\right) \\
& +Q_{\mathrm{L}} \frac{\left(\mathbf{r}-\mathbf{R}_{\mathrm{L}}, \delta \mathbf{R}_{\mathrm{XL}}\right)}{\left|\mathbf{r}-\mathbf{R}_{\mathrm{L}}\right|^{3}}
\end{aligned}
$$

The vectors involved in the above formula refer to the complex geometry and its variation:

$$
\mathbf{R}_{\mathrm{M}}=(0,0,0) \quad \mathbf{R}_{\mathrm{L}}=\left(0,0, r_{\mathrm{ML}}\right) \quad \delta \mathbf{R}_{\mathrm{XL}}=\left(0,0, \delta r_{\mathrm{XL}}\right) .
$$

Applying the standard formulae of electrostatics we can write the extra field exerted at the point $r$ due to the above potential variation Eq. (3):

$$
\begin{gathered}
\delta \mathrm{E}_{\text {Coul }}(\mathbf{r})=-\nabla \delta \varphi_{\text {Coul }}(\mathbf{r}) \\
=-\delta Q_{\mathrm{XL}}\left(\frac{\mathbf{r}-\mathbf{R}_{\mathrm{L}}}{\left|\mathbf{r}-\mathbf{R}_{\mathrm{L}}\right|^{3}}-\frac{\mathbf{r}-\mathbf{R}_{\mathrm{M}}}{\mid \mathbf{r}-\mathbf{R}_{\left.\mathrm{M}\right|^{3}}}\right)-Q_{\mathrm{L}} \frac{\delta \mathbf{R}_{\mathrm{XL}}}{\left|\mathbf{r}-\mathbf{R}_{\mathrm{L}}\right|^{3}} \\
\quad+Q_{\mathrm{L}} \frac{\left(\mathbf{r}-\mathbf{R}_{\mathrm{L}}, \delta \mathbf{R}_{\mathrm{XL}}\right)\left(\mathbf{r}-\mathbf{R}_{\mathrm{L}}\right)}{\mid \mathbf{r}-\mathbf{R}_{\left.\mathrm{L}\right|^{5}}}
\end{gathered}
$$

The ideal position for the trans-ligand $\mathrm{L}$ is:

$$
\mathbf{r}=\mathbf{R}_{\mathrm{L}}^{\mathrm{trans}}=\left(0,0,-r_{\mathrm{ML}}\right)
$$

and the electrostatic force additional to that it had to experience in the $\mathrm{ML}_{6}$ complex reads:

$$
\delta \mathbf{f}_{\text {Coul }}\left(\mathbf{R}_{\mathrm{L}}^{\text {trans }}\right)=\frac{1}{4} \frac{Q_{\mathrm{L}}^{2}}{r_{\mathrm{ML}}^{2}}\left(\frac{\delta r_{\mathrm{XL}}}{r_{\mathrm{ML}}}+3 \frac{\delta Q_{\mathrm{XL}}}{Q_{\mathrm{L}}}\right)(0,0,1) .
$$

Then according to approximation given by Eq. (1) one can conclude that since the force felt by the trans-ligand in the substituted molecule differs by $\delta f_{\text {Coul }}\left(\mathbf{R}_{\mathrm{L}}^{\text {trans }}\right)$ the coordinate of the trans-ligand differs by the same force divided by the elasticity constant characteristic for the nuclear shift along the given direction. Incidentally the required elasticity constant equals to the elasticity constant for the ML bond stretching in the classical MM force field, so that:

$$
\delta \mathbf{R}_{\mathrm{L}}^{\text {trans }}=\frac{\delta \mathbf{f}_{\mathrm{Coul}}\left(\mathbf{R}_{\mathrm{L}}^{\text {trans }}\right)}{K_{\mathrm{ML}}} .
$$

This result can be qualitatively understood as follows: two factors contribute to the trans-influence. First is the charge redistribution. Indeed, if for the substituent ligand $X$ the effective charge decreases by its absolute value (the negative $Q_{\mathrm{L}}$ becomes less negative, i.e., $\delta Q_{\mathrm{XL}}>0$ ) this results in an effective attractive force. However, if for the substituent ligand $X$ its effective charge increases by absolute value as compared to that of the ligand $L$ (the negative $Q_{L}$ becomes more negative, i.e., $\delta Q_{X L}<0$ ) this contributes an effective repulsive force acting on the trans-ligand. Nevertheless, due to the assumption that the charge brought to the substituent $X$ is taken from the central atom $M$ the latter acquires additional charge $-\delta Q_{\mathrm{XL}}$, which is located much closer to the trans-ligand than the substituent and produces a stronger force. The overall effect of the charge redistribution is opposite to that exerted by the charge transferred to the substituent and it is the trans-ML bond contraction for the more negative substituent ligand $X$ and the trans-ML bond elongation for the less negative substituent ligand $X$. Even this already rather complicated picture can significantly change since the net effect depends also on the geometry shift of the substituent ligand $X$ relative to the ideal position of the ligand $L$. If $\delta r_{\mathrm{XL}}>0$ (the substituent is further away from the central atom) the repulsion of the trans-ligand from the substituent decreases, which results in an effective attraction force acting on the trans-ligand and by this reducing the ML bond length. The overall trans-influence in the Coulomb approximation is controlled by the quantity

$$
\frac{\delta r_{\mathrm{XL}}}{r_{\mathrm{ML}}}+3 \frac{\delta Q_{\mathrm{XL}}}{Q_{\mathrm{L}}}
$$


If it is positive the substitution results in an effective attraction of the trans-ligand (and thus in the shortening of the bond in the trans-position to the substituent) and vice versa.

The situation with the ligands in the cis-position to the substituent is even more complicated. Taking for the sake of definiteness the cis-ligand at

$$
\mathbf{r}=\mathbf{R}_{\mathrm{L}}^{\mathrm{cis}}=\left(r_{\mathrm{ML}}, 0,0\right)
$$

which is one of the four equivalent positions we see that the additional electrostatic force acting on it is:

$$
\begin{aligned}
& \delta \mathbf{f}_{\mathrm{Coul}}\left(\mathbf{R}_{\mathrm{L}}^{\mathrm{cis}}\right) \\
& \quad=\frac{1}{2 \sqrt{2}} \frac{Q_{\mathrm{L}}^{2}}{r_{\mathrm{ML}}^{2}}\left(\frac{\delta r_{\mathrm{XL}}}{r_{\mathrm{ML}}}\left(\frac{-3}{2}, 0, \frac{1}{2}\right)+\frac{\delta \mathrm{Q}_{\mathrm{XL}}}{Q_{\mathrm{L}}}(1-2 \sqrt{2}, 0,1)\right)
\end{aligned}
$$

which (depending on the relation between $\delta \mathrm{rXL} /$ $r \mathrm{ML}$ and $\delta \mathrm{QXL} / Q \mathrm{Q}$ ) may result either in approach of the cis-ligand to the central atom of the complex or in its going further from the latter. The elongation of the apical bond $\left(\delta r_{\mathrm{XL}}>0\right)$ always tends to shorten the bond in the cis-position to it. On the other hand, the substitution by a less electronegative substituent $\left(\delta Q_{\mathrm{XL}}>0\right)$ results in an effective repulsion of the cis-ligand (lengthening of the bond in the cis-position to the substituent). But this effect is expected to be somewhat less pronounced than the bond-length variation for the trans-ligand:

$$
\frac{\left|\delta \mathbf{f}_{\text {Coul }}\left(\mathbf{R}_{\mathrm{L}}^{\text {cis }}\right)\right|}{\left|\delta \mathbf{f}_{\text {Coul }}\left(\mathbf{R}_{\mathrm{L}}^{\text {trans }}\right)\right|}=\frac{4}{3} \frac{2 \sqrt{2}-1}{2 \sqrt{2}} \approx 0.86<1
$$

although the difference should not be very significant (only 15\%). In all cases (which are expectable) there is a component of the force acting along the shift direction of the substituent $X$.

\section{VAN DER WAALS CONTRIBUTION TO LIGAND INFLUENCE}

Although one may think that on the scale of the Coulomb interactions the effect of other (van der Waals) nonbonding interactions may be neglected we notice that in the present context it goes not about the absolute magnitude of the forces exerted by different nonbonding components of the classical force fields upon the idealized structure of the complex but about the variations of the forces exerted by these fields. With this precaution in mind we address the effect of monosubstitution upon the forces exerted by the substituent within the simplest possible model of the van der Waals force field-the Lennard-Jones [6-12] potential. In this approximation the contribution to the potential energy of the vdW interaction of a particle $L$ at the point $r$ with the particle at $R_{X}$ has the form:

$$
V_{\mathrm{LX}}(\mathbf{r})=\varepsilon_{\mathrm{LX}}\left(\tilde{y}^{2}-2 \tilde{y}\right)
$$

where

$$
\tilde{y}=\left(\frac{d_{\mathrm{LX}}}{\left|\mathbf{r}-\mathbf{R}_{\mathrm{X}}\right|}\right)^{6}
$$

In this notation the minimum of the LJ potential curve corresponds to $\tilde{y}=1$. Using the Berthelot combination rules we get for the substituted case:

$$
\begin{gathered}
\varepsilon_{\mathrm{XL}}=\sqrt{\varepsilon_{\mathrm{LL}} \varepsilon_{\mathrm{XX}}}=\sqrt{\varepsilon_{\mathrm{LL}}\left(\varepsilon_{\mathrm{LL}}+\delta \varepsilon_{\mathrm{XL}}\right)} \approx \varepsilon_{\mathrm{LL}}\left(1+\frac{1}{2} \frac{\delta \varepsilon_{\mathrm{XL}}}{\varepsilon_{\mathrm{LL}}}\right) \\
d_{\mathrm{XL}}=\frac{1}{2}\left(d_{\mathrm{LL}}+d_{\mathrm{XX}}\right)=d_{\mathrm{LL}}+\frac{1}{2} \delta d_{\mathrm{XL}} .
\end{gathered}
$$

Inserting the linear approximation for the distance between the point $r$ and the position of the substituent $R_{X}$ like in the case of the Coulomb interaction:

$$
\begin{aligned}
\left|\mathbf{r}-\mathbf{R}_{\mathrm{X}[\mathrm{b}]}\right|=\mid \mathrm{r} & -\mathrm{R}_{\mathrm{L}}-\delta \mathbf{R}_{\mathrm{XL}} \mid \\
& \approx\left|\mathbf{r}-\mathbf{R}_{\mathrm{X}}\right|-2\left(\mathbf{r}-\mathbf{R}_{\mathrm{L}}, \delta \mathbf{R}_{\mathrm{XL}}\right)
\end{aligned}
$$

and performing somewhat long but simple algebra we arrive to an estimate of the substitution stipulated variation of the potential energy of particle L located in the point $r$ :

$$
\begin{aligned}
\delta V_{\mathrm{vdW}}(\mathbf{r}) & \approx \frac{1}{2} \delta \varepsilon_{\mathrm{XL}}\left(y^{2}-2 y\right) \\
+ & 12 \varepsilon_{\mathrm{LL}}\left(\frac{\delta d_{\mathrm{XL}}}{d_{\mathrm{LL}}}+\frac{\left(\mathbf{r}-\mathbf{R}_{\mathrm{L}} \delta \mathbf{R}_{\mathrm{XL}}\right)}{\left|\mathbf{r}-\mathbf{R}_{\mathrm{L}}\right|^{2}}\right)\left(y^{2}-y\right)
\end{aligned}
$$

where

$$
y=\left(\frac{d_{\mathrm{LL}}}{\left|\mathbf{r}-\mathbf{R}_{\mathrm{L}}\right|}\right)^{6}
$$

The additional forces have the form: 


$$
\begin{gathered}
\delta \mathbf{f}_{\mathrm{vdW}}(\mathbf{r})=-\nabla \delta V_{\mathrm{vdW}}(\mathbf{r})=-\delta \varepsilon_{\mathrm{XL}}(y-1) \nabla y- \\
-12 \varepsilon_{\mathrm{LL}}\left(y^{2}-y\right) \nabla \frac{\left(\mathbf{r}-\mathbf{R}_{\mathrm{L}}, \delta \mathbf{R}_{\mathrm{XL}}\right)}{\left|\mathbf{r}-\mathbf{R}_{\mathrm{L}}\right|^{2}}- \\
-12 \varepsilon_{\mathrm{LL}}\left(\frac{\delta d_{\mathrm{XL}}}{d_{\mathrm{LL}}}+\frac{\left(\mathbf{r}-\mathbf{R}_{\mathrm{L}}, \delta \mathbf{R}_{\mathrm{XL}}\right)}{\left|\mathbf{r}-\mathbf{R}_{\mathrm{L}}\right|^{2}}\right)(2 y-1) \nabla y .
\end{gathered}
$$

Performing necessary algebra yields rather cumbersome formulae (not given here) which, however, allow for the qualitative understanding. Like in the case of the Coulomb interaction the additional forces in the case of the van der Waals interaction appear from two sources-the variation of the parameters of the Lennard-Jones (LJ) potential and the variation of the idealized geometry. In the accepted first order approximation these two sources contribute independently. The variations of the potential parameters $\left(\delta \varepsilon_{\mathrm{XL}}\right.$ and $\left.\delta d_{\mathrm{XL}}\right)$ are even more uncertain than the values of $\varepsilon_{\mathrm{LL}}$ and $d_{\mathrm{LL}}$ themselves. If one neglects their contribution the result can be understood from the analysis of the LJ potential curve. For the trans-ligand two situations are thinkable: one is that the ideal L-L separation (equal to $2 r_{\mathrm{ML}}$ ) falls onto the repulsive segment of the LJ curve $\left(\mathrm{d}_{\mathrm{LL}} / 2 r_{\mathrm{ML}}<1\right)$. Then, getting the $\mathrm{M}-\mathrm{X}$ bond longer than the $\mathrm{M}-\mathrm{L}$ bond results in abstraction of the repulsive wall from the trans L ligand, which manifests itself in the effective attraction and in the shortening of the trans-bond. However, if the transligand appears on the attractive segment of the LJ curve $\left(\mathrm{d}_{\mathrm{LL}} / 2 r_{\mathrm{ML}}>1\right)$ the situation is not that simple. For shorter distances (closer to the potential minimum) the restoring force (the first derivative of the potential) increases when the interatomic separation increases. In this case getting the $\mathrm{M}-\mathrm{X}$ bond longer than the $\mathrm{M}-\mathrm{L}$ bond results in the increase of the restoring force and thus to an effective extra attraction of the trans L ligand. This has to result in a shortening of the trans $\mathrm{M}-\mathrm{L}$ bond as well. On the other hand, the restoring force on the attractive segment passes through its maximum since at larger distances it decreases and completely vanishes at the infinite separation. The larger and shorter distance ranges on the attractive segment of the LJ potential are separated by the value:

$$
y^{*}=\frac{7}{13}
$$

If $y<y^{*}$ the elongation of the $M-X$ bond as compared to the $\mathrm{M}-\mathrm{L}$ bond results in the decrease of the restoring force and thus to an effective extra repulsion of the trans L ligand.

The situation with the cis-ligands is even more complicated. Performing a shift of the $\mathrm{X}$ ligand as compared to the original position of the L ligand produces additional force acting on the cis-ligand, which has two components: one directed along the $x$-axis of the complex coordinate frame, and that directed along the $z$-axis. Both of these contributions may be either of attractive or repulsive character, i.e., the $x$-component of the force may be directed either to the central atom or out of it, whereas the $z$-component of the force may be pointing to the same direction as the shift of the $X$ ligand relative to the ideal position of the L ligand. The actual direction of the extra force depends on the precise position of the cis-ligand relative to the substituted one. For example, for the more distant $X$ ligand, the $y$-component of the force is directed away from the central atom for $y<y^{*}=4 / 7$ and toward it for shorter distances meaning larger values of the variable $y$. On the other hand the $z$ component of the force is directed opposite to the shift direction of the $X$ ligand for $y<y^{* *}=1 / 2$.

\section{LIGAND INFLUENCE AS DERIVED FROM NONBONDING FORCE FIELDS VS. EXPERIMENT}

From the above treatment one can derive two conclusions: (1) The diversity of the types of behavior which in principle can be ascribed to different nonbonding force fields is impressive. By assigning these or those values to the parameters characterizing the force field variation under substitution one can reproduce eventually all thinkable modes of mutual influence of the ligands in the coordination sphere. (2) The parameters' values possibly needed to reproduce the observed behavior cannot be systematized and their values will probably remain the result of a play of uncontrollable factors. This reduces the validity of the entire picture since certain type of systematization is possible in chemical terms (see later). Here we are going to discuss it briefly relying basically upon the review given in Ref. [8], which will be compared with the above theoretical sketches.

The ligand influence in coordination compounds of nonmetals in higher and lower oxidation states differs significantly both in magnitudes and signs of the effects. An instructive example is provided by the substitution in the perhalogenated com- 
plexes which can be described as $\mathrm{L}_{6} \mathrm{M} \rightarrow \mathrm{L}_{5} \mathrm{XM}$ where the role of $\mathrm{L}$ is taken by a halogen anion, $\mathrm{X}$ stands for a ligand less electronegative than the halogen $\mathrm{L}$, and $\mathrm{M}$ stands for a nontransition element like J, S, P, Sn, etc., in a higher oxidation state. In some cases the substitution does not cause any difference between the cis- and trans-bond-lengths or only a marginal one (compounds of S(VI)). However, in other cases the substitution results in remarkable deformations of the coordination octahedron (compounds of $\mathrm{J}(\mathrm{VII})$ ). In the latter case the cis-J-F bonds are significantly shorter than the J-F bond in the trans-position to the presumably less negatively charged oxygen substituent. This situation can be characterized by some values $\delta Q_{\mathrm{XL}}>0$, $Q_{\mathrm{L}}<0$, and $\delta r_{\mathrm{XL}}<0$, so that the entire picture qualitatively fairly agrees with the Coulomb model of trans-effect described in the section Coulomb Contribution to Ligand Influence. Turning to the beginning of the Periodic row one can see that namely the bond in the trans-position to the substituent turns out to be significantly shorter than those in the cis-positions for the complexes of $\mathrm{P}(\mathrm{V})$, $\mathrm{As}(\mathrm{V}), \mathrm{Sn}(\mathrm{IV}), \mathrm{Pb}(\mathrm{IV}), \mathrm{Ga}(\mathrm{III})$. Although in all these cases one could expect a significant contribution of the charge redistribution effect upon the complex geometry through the mediation of the Coulomb forces, the picture is inverted as compared to the predictions based on the Coulomb contributions to the MM energy (and to the case of J(VII) complexes). Remarkably enough that this contradiction most probably cannot be cured by referring to the bond length variations $\delta r_{\mathrm{XL}}$, which are mostly negative since one can expect that the XM bonds in the examples reviewed in Ref. [8] are shorter than the LM ones.

On the other hand, in the case of complexes with nonmetallic central atom of a lower oxidation degree the electropositive substitution (in complexes of $\mathrm{Te}(\mathrm{IV})$ and $\mathrm{Se}(\mathrm{IV}))$ results in a significant increase of the bond-length in the trans-position to the substitution as compared to the lengths of the cisbonds. Similar picture is observed in the coordination compounds of transition metals. So we see that the diversity of the observed types of behavior is too large to try to squeeze the available experimental data to the picture relying upon the Coulomb forces acting between the effective charges of restricted mobility. Doing so one drives in the situation when the demands to the relative magnitudes of the charges' and ideal bond-lengths' variations $\left(\delta \mathrm{r}_{\mathrm{XL}} / r_{\mathrm{ML}}\right.$ and $\left.\delta \mathrm{Q}_{\mathrm{XL}} / Q_{\mathrm{L}}\right)$ become very difficult to satisfy. Much more important is that the quantities $\delta Q_{\mathrm{XL}}$ playing a key role in the picture of the ligand influence based on the Coulomb forces as the bare charges $Q_{\mathrm{L}}$ themselves must be found from some kind of quantum chemically based procedure since otherwise one cannot guarantee the correct behavior of them. At the same time any attempt to reload the specific behavior observed in different complexes upon the parameters of the LJ potentials brings up a necessity to assume the dependence of these parameters on the nature of the "third"-central atom, which is clearly not desirable. One can try to get around these problems by ascribing the values of different signs to the off-diagonal stretchingstretching constants when it goes about the cis- and trans-bonds. The signs of these constants may be made different depending on the nature (and the oxidation state) of the central atom. Thus the necessary values will have to be assigned on the basis of more and more refined system of atomic types as it is done in the "classical" MM, but this brings back the problem of enormous growth of the number of necessary parameters and of setting physically sound limits to their values. Our purpose in the present paper is ultimately to sequentially derive the expression for the required off-diagonal terms and to obtain estimates for their values. This will be done in further sections on the basis of analysis of interrelations between the perturbations of the electronic structure of the coordination compound and its geometry, having the theory of the ligand influence by Levin and Dyachkov (which successfully described the cases reviewed above) as a benchmark to be reached by the scheme under construction.

\section{Account of Deductive Molecular Mechanics}

\section{GENERAL SETTING}

The methodology designed for deriving mechanistic picture of PES from one based on a suitable quantum description of molecular electronic structure has been proposed in Ref. [12] under the name of deductive molecular mechanics (DMM). It has been applied in Refs. [13-15] to the "organic" molecules-the main object of the classical MM with a considerable success. The result of these works summarized in Ref. [16] is that a sequential derivation of whatever mechanistic model of PES for each 
specific class of molecules consists of the following steps:

- Groups of electrons responsible for the observed features of molecular energy and geometry to be reproduced in the target MM model must be identified.

- Approximate methods sufficient for description of the responsible electron groups and reproducing the necessary features must be identified and formalized in the structure of the trial wave function for the target class of molecules.

- The electronic structure variables (ESVs) describing the relevant electron groups in the sufficient approximations must be identified.

- In terms of these sets of ESVs one constructs an intermediate (DMM) model of the PES.

- The intermediate ESVs can be excluded, for example, using a linear response theory in order to get the target MM model of PES.

For the molecules of interest in the present paper-the complexes of nontransition elements the results first three stages can be extracted by analyzing some previous works. As we mentioned previously the LD theory serves as a benchmark here since it allows to establish and by comparison with experiment to verify relations between some elements of molecular electronic structure and molecular geometry of coordination compounds.

\section{CONSTRUCTING DMM FOR OCTAHEDRAL COMPLEXES}

As any phenomenological theory the LD theory of the ligand influence tacitly assumes the existence of an effective Hamiltonian describing certain group of electrons responsible for the experimentally observed behavior. The orbitals responsible for the binding of the central atom with donor ligands can be reasonably identified with the valence AOs of the corresponding central atoms and with the hybrid orbitals (HOs) of the lone pairs (LPs) of the ligand donor atoms. These orbitals and electrons residing in the central atom and in the closest vicinity of the latter can be termed as the CLS. In their terms the explanation of experimental behavior has been given in Ref. [8]. The problem is how to sequentially define the orbitals to be used to span the CLS in a polyatomic system like a coordination compound with organic ligands. Paper [17] gives a tentative answer to this question. There we performed comparative study of electronic structures of simple amines and ethers on one hand and of their polycyclic counterparts on another hand by the semiempirical SLG-MNDO method $[18,19]$. The results given in Ref. [17] show that the relevant characteristics of electronic structure (the bond orders, electron densities on the bonding orbitals of the donor atoms, and the weights of the s-functions in the LPs) of the low-molecular amines and ethers and their polycyclic analogs are fairly close. For this reason we can assume that the LP HOs required for the CLS construction can be taken from some the strictly local geminal (SLG) based procedure for free ligands and are subsequently only slightly modified due to complexation.

Next question to be answered relates to the form of the wave function $\Phi_{\text {CLS }}$ of the group of electrons in the carrier space defined above and to the acceptable approximation to be used for obtaining it. The LD theory of ligand influence had been constructed with use of the Hückel type procedure. By this the Coulomb interaction within the considered subset of oneelectron states was not taken into account although (as it is shown in the section Coulomb Contribution to Ligand Influence) it affects the process of the charge (electron density) redistribution in the CLS and can give a considerable contribution to the energy (see later). The HFR aproximation takes into account the necessary Coulomb terms and by this the true energy operator for the CLS group is the effective Fock operator. This reduces the problem to solving the system of Hartree-Fock equations for the occupied and vacant MOs in the carrier space of the CLS electron group. For the case of an octahedral complex the problem is further considerably simplified by symmetry. Only the following symmetry adapted linear combinations first introduced in Refs. [20-22] are allowed to serve as either occupied or vacant canonical MOs of the CLS:

$$
\begin{aligned}
\psi\left(e_{g} c\right)= & \frac{1}{\sqrt{12}}\left(2 \chi_{z}+2 \chi_{-z}-\chi_{x}-\chi_{-x}-\chi_{y}-\chi_{-y}\right) \\
\psi\left(e_{g} s\right) & =\frac{1}{2}\left(\chi_{x}+\chi_{-x}-\chi_{y}-\chi_{-y}\right) \\
\psi^{a}\left(a_{1 g}\right)= & -x_{a_{19}} \phi_{s}+\frac{y_{a_{1 g}}}{\sqrt{6}} \\
& \times\left(\chi_{x}+\chi_{y}+\chi_{z}+\chi_{-x}+\chi_{-y}+X_{-z}\right)
\end{aligned}
$$




$$
\begin{aligned}
\psi^{b}\left(a_{1 g}\right)=y_{a_{1 g}} \phi_{s} & +\frac{x_{a_{1 g}}}{\sqrt{6}} \\
& \times\left(\chi_{x}+\chi_{y}+\chi_{z}+\chi_{-x}+\chi_{-y}+\chi_{-z}\right) \\
\psi^{a}\left(t_{1 u} \gamma\right) & =-x_{t_{1 u}} \phi_{\gamma}+\frac{y_{t_{1 u}}}{\sqrt{2}}\left(\chi_{\gamma}-\chi_{-\gamma}\right) \\
\psi^{b}\left(t_{1 u} \gamma\right) & =y_{t_{1 u}} \phi_{\gamma}+\frac{x_{t_{1 u}}}{\sqrt{2}}\left(\chi_{\gamma}-\chi_{-\gamma}\right)
\end{aligned}
$$

where the superscripts $a$ and/or $b$ refer to the antibonding or bonding linear combination of the symmetry $\Gamma \gamma$ (here $\Gamma$ stands for the irreducible representation and $\gamma$ for its row). The basis functions $\phi$ are the one-electron states $\phi_{s}$-the s-orbital of the central atom and $\phi_{\gamma}(\gamma=x, y, z)$-three $p$-orbitals of the latter directed long the coordinate axes, and the functions $\chi_{\gamma^{\prime}} \chi_{-\gamma}$ are the LP HOs directed along the $\gamma$-axis $(\gamma=x, y, z)$ of the Cartesian coordinate system centered, respectively, at the donor atom of the ligand located on the positive and negative semiaxes $\gamma$. There is only one instance of the MO transforming according to either row (c or $s$ ) of the $e_{g}$ symmetry. In the 12-electron complexes (in the present context it goes about the number of electrons in the CLS electron group), which will be considered below, they are occupied. Coefficients $x_{\Gamma}$ and $y_{\Gamma}=\sqrt{1-x_{\Gamma}^{2}}$ describe the mixing between the central ion AOs and the ligand HOs and are to be determined from the secular equations of the SCF MO LCAO method. This all reduces the number of the variables describing the electronic structure of the octahedral complexes (its CLS) to only two, which contain all information necessary to describe the octahedral CLS, e.g.: $x_{a_{1 g}}$ and $x_{t_{11}}$. In the octahedral symmetry the orbitals of each Ã $\gamma$ appear no more than twice. For that reason the problem of defining variables $x_{a_{1}}$ and $x_{t_{1 u}}$ (or their equivalentssee later) reduces to diagonalization of the $2 \times 2$ Fockian blocks corresponding to the respective irreducible representations:

$$
F^{\Gamma}=\left(\begin{array}{ll}
a_{\Gamma} & b_{\Gamma} \\
b_{\Gamma} & c_{\Gamma}
\end{array}\right)
$$

The exact definition of the matrix elements of the Fockian for an SCF-treated group of electrons in the presence of other groups is given in Refs. [17, 23].

The one-electron density matrix corresponding to the solution of the Hartree-Fock problem in the
CLS is as any Hartree-Fock density matrix an operator (matrix) $P$ projecting to the occupied MOs:

$$
\begin{aligned}
& \left.P=x_{a_{1}}^{2}\left|a_{1}^{0}\right\rangle\left\langle a_{1}^{0}\left|+y_{a_{1}}^{2}\right| s\right\rangle\left\langle s\left|+x_{a_{1}} y_{a_{1}}\right| s\right\rangle\left\langle a_{1}^{0}|+| a_{1}^{0}\right\rangle\langle s|\right) \\
& +\sum_{\gamma=c, s}\left|e_{g}^{0} \gamma\right\rangle\left\langle e_{g}^{0} \gamma\right|++\sum_{\gamma=x, y, z}\left[x_{t_{1 u}}^{2}\left|t_{1 u}^{0} \gamma\right\rangle\left\langle t_{1 u}^{0} \gamma\left|+y_{t_{1 u}}^{2}\right| p_{\gamma}\right\rangle\right. \\
& \left.\quad \times\left\langle p_{\gamma}\right|+x_{t_{1 u}} y_{t_{1 u}}\left(\left|p_{\gamma}\right\rangle\left\langle t_{1 u}^{0} \gamma|+| t_{1 u}^{0} \gamma\right\rangle\left\langle p_{\gamma}\right|\right)\right]
\end{aligned}
$$

where the quantities $x_{\Gamma}, y_{\Gamma}$ are defined after Eq. (17) and the orbitals with the superscript " 0 " refer to the symmetry adapted combinations of the LP HOs $\chi_{\gamma}$ in the right hand side of Eq. (13). The above expression can be further simplified by noticing that the normalization condition for the quantities $x_{\Gamma}, y_{\Gamma}$ can be absorbed in a rational function of another (single) ESV for each $\Gamma$. Indeed, a two-dimensional operator projecting onto one-dimensional subspace has the form:

$$
P_{\Gamma}=\left(\begin{array}{cc}
x_{\Gamma}^{2} & x_{\Gamma} y_{\Gamma} \\
x_{\Gamma} y_{\Gamma} & y_{\Gamma}^{2}
\end{array}\right)=\frac{1}{1+v_{\Gamma}^{2}}\left(\begin{array}{cc}
1 & v_{\Gamma} \\
v_{\Gamma} & v_{\Gamma}^{2}
\end{array}\right)
$$

The projection operator Eq. (15) is a direct sum of the $2 \times 2$ projectors with the appropriate values of $v_{\Gamma}$ (in particular $v_{e_{g}}=0$ ) taken in the required number of instances (one for each row of the irreducible representation $\Gamma$ ). The projection operator Eq. (15) is one for the 12-electron complex. If it goes about a 14-electron complex the $P_{a_{1}}$ in the direct sum has to be replaced by the $2 \times 2$ identity matrix, thus reducing the number of ECVs to only one: $v_{t_{11}}$.

Inserting the ground state projection operator in the Hartree-Fock expression for the energy of the CLS electron group we get:

$$
\begin{aligned}
E_{\mathrm{CLS}}=\left(2 \mathrm{Sp} h^{\mathrm{eff}} P+\operatorname{Sp} P \Sigma[P]\right), \quad \text { provided } \\
F_{\mathrm{CLS}}=h^{\mathrm{eff}}+\Sigma[P],
\end{aligned}
$$

where $h^{\text {eff }}$ is the one-electron part of the Fock operator and $\Sigma[P]$ is the self-energy part representing the electrostatic field induced by electrons in the CLS group upon each other. We arrive to an explicit expression for the energy in terms of the ESVs $v_{\Gamma}$. This is the closed expression for the energy required by the DMM methodology (the molecular geometry enters through the respective dependence of the Fockian matrix elements). Moreover it is rational function of the ESVs involved. This expression can be efficiently searched for minimum with respect to the relevant variables, yielding the 
equilibrium geometry and corresponding electronic structure. For example the effective charges-key quantities for the considerations of the section Coulomb Contribution to Ligand Influence, appear as averages of $P$ over the corresponding AOs or HOs.

It is possible, however, to obtain analytical estimates for the equilibrium values of ESVs, which possess rather interesting properties. The simplest analytical expression representing the solution can be written for the product $x_{\Gamma} y_{\Gamma}$, which is expressed through the single parameter $\zeta_{\Gamma}$ :

$$
\zeta_{\Gamma}=\frac{b_{\Gamma}}{c_{\Gamma}-a_{\Gamma}}
$$

condensing all the necessary information:

$$
x_{\Gamma}^{2} y_{\Gamma}^{2}=\frac{1}{4}\left(1-\frac{1}{1+\zeta_{\Gamma}^{2}}\right) .
$$

If one is interested in the complex formation then the limit $\zeta_{\Gamma} \ll 1$ has to be considered. In this case:

$$
x_{\Gamma}^{2} y_{\Gamma}^{2} \approx \frac{1}{4} \zeta_{\Gamma}^{2}
$$

The opposite limit $\zeta_{\Gamma} \gg 1$ describes the situation close to the equilibrium. In this case the following estimate holds:

$$
x_{\Gamma}^{2} y_{\Gamma}^{2} \approx \frac{1}{4}\left(1-\frac{1}{\zeta_{\Gamma}^{2}}\right)
$$

These results for surely known for decades as far as we know have never been considered from the point of view of possible transferability of the offdiagonal density among different molecules. This latter property is however a key to constructing any mechanistic model of PES as it is shown in Ref. [16].

The situation described by the formula Eq. (18) differs in an important respect from analogous results of Ref. [15] proven for isolated two-center two-electron bonds characteristic to organic species. In the "organic" domain the transferability of the off-diagonal element of the one-electron density matrix immediately brought up the transferability of the corresponding Coulson bond-order directly involved in the expression for the bond energy. The formula Eq. (18), however, applies to the density matrix element in the basis of the symmetry adapted linear combinations of the LP HOs. They are not related to individual $\mathrm{M}-\mathrm{L}$ bonds, which are not even "observable" elements of molecular electronic structure in the sense proposed by Ruedenberg [24] (in opposition with the two-center two-electron bonds in "organics"). By contrast the stable (upto the second order in the presumably small parameters $\zeta_{\Gamma}^{-1}$ ) values of the one-electron density matrix elements refer to a completely different elements of the construction: to the threedimensionally delocalized CLS group of electrons whose ESVs themselves possess necessary transferability properties, which makes it an "observable" component of the molecular electronic structure in the sense of Ref. [24]. Pragmatic outcome of this might be in replacing in the vicinity of equilibrium of the ESVs either by transferable value of $v_{\Gamma}=1$ $\left(\Gamma=a_{1}, t_{1 u}\right)$ or by inserting the estimates Eq. (18) and by this arriving to the PES as a function of the nuclear coordinates only. The described result applies however to the octahedral complexes only. The major task is to extend this treatment to the complexes of lower symmetry, which will be done in subsequent sections.

\section{Perturbative Analysis of the DMM Model of CLS and Its Relation to LD Theory of Ligand Influence}

Now let us consider what is going to happen to the above DMM picture under the variation of composition (chemical substitution) and/or geometry both reducing the symmetry of the CLS. Interplay between these two types of perturbation was the main concern in the LD theory of ligand influence. This theory evolves in terms of two key objects: the substitution operator and the electron-vibration (vibronic) interaction operator. These two perturbations are applied to the matrix representation of the CLS Fockian written with respect to formally the same set of one-electron states (central atom AOs and LP HOs). In this setting the dependence of the Fockian on the chemical composition of the species involved reduces to the corresponding dependence of its matrix elements. Analogously the geometry dependence becomes that of the matrix elements of the Fockian.

\section{DMM ON NONSYMMETRICAL COORDINATION COMPOUNDS}

Whatever Fock operator can be represented as a symmetric one and a perturbation of the latter, 
which includes both the dependence of the matrix elements on nuclear shifts from the equilibrium positions and the transition to a less symmetric environment due to the substitution. To pursue this way employing the above ESVs we introduce first some notations. Let $h^{\prime}$ be the supervector of the first derivatives of the matrix of the Fock operator with respect to nuclear shifts $\delta q$ counted from a symmetrical equilibrium configuration. By a supervector we understand here a vector whose components numbered by the specific nuclear shifts are themselves $10 \times 10$ matrices of the first derivatives of the Fock operator with respect to the latter. Then the scalar product of the vector of all nuclear shifts $\delta q$ ) and of the supervector $h^{\prime}$ yields a $10 \times 10$ matrix of the corrections to the Fockian linear in the nuclear shifts:

$$
\left(h^{\prime} \mid \delta q\right)=\sum_{i} \frac{\partial h}{\partial q_{i}} \delta q_{i}
$$

Next, let $h^{\prime \prime}$ be the supermatrix of the second derivatives of the matrix of the Fock operator with respect to the same shifts. As previously we refer here to supermatrix indexed by the pairs of nuclear shifts in order to stress that the elements of this matrix are themselves the $10 \times 10$ matrices of the corresponding second derivatives of the Fockian with respect to the shifts. The contribution of the second order in the nuclear shifts can be given the form of the (super)matrix average over the vector of the nuclear shifts:

$$
\left(\delta q\left|h^{\prime \prime}\right| \delta q\right)=\sum_{i j} \frac{\partial^{2} h}{\partial q_{i} \partial q_{j}} \delta q_{i} \delta q_{j}
$$

Supplying this all with the $10 \times 10$ matrix of the substitution operator

$$
h^{S}=F_{S}^{\mathrm{CLS}}=F_{\mathrm{ML}_{\mathrm{n}} X Y Z}^{\mathrm{CLS}} \ldots-F_{\mathrm{ML}_{6}}^{\mathrm{CLS}}
$$

we get the "bare" perturbation of the effective Fockian in the CLS carrier space as:

$$
\left(h^{\prime} \mid \delta q\right)+\frac{1}{2}\left(\delta q\left|h^{\prime \prime}\right| \delta q\right)+h^{S}
$$

This does not form the entire ("dressed") perturbation since in case the electron density changes to the first order in the above perturbation the Fockian acquires additional perturbation through the variation of its self-energy part, which leads to the self-consistent perturbation. Thus the perturbed Fockian can be written as:

$$
F=F_{0}\left[P_{0}\right]+\left(h^{\prime} \mid \delta q\right)+\frac{1}{2}\left(\delta q\left|h^{\prime \prime}\right| \delta q\right)+h^{S}+\Sigma[\Delta P]
$$

Here $\Delta P$ stands for the correction to the unperturbed projection operator $P_{0}$ to the occupied MOs, which in case to the octahedral complexes equals to $P$ given by Eq. (15). This serves as a prerequisite for performing two remaining steps of the recipe of section Account of Deductive Molecular Mechanics of constructing a DMM description of coordination compounds of arbitrary (low) symmetry and of the linear response theory based on it and leading to strictly mechanistic description of this class of compounds.

To proceed further we look what is the perturbed density matrix. It was assumed to have the form

$$
P=P_{0}+\Delta P=P_{0}+\sum_{n>0} P^{(n)}
$$

where the correction $\Delta P$ can be expanded in terms of the matrices $V$ satisfying the conditions:

$$
\begin{aligned}
& P_{0} V=0 ; \quad V P_{0}=V ; \quad\left(1-P_{0}\right) V P_{0}=V ; \\
& P_{0} V^{+}=V^{+} ; \quad P_{0} V^{+}\left(1-P_{0}\right)=V^{+} ; \quad V^{+} P_{0}=0
\end{aligned}
$$

as follows [25]:

$$
\begin{array}{r}
P^{(1)}=V+V^{+}, \quad P^{(2)}=V V^{+}-V^{+} V, \\
P^{(3)}=-V V^{+} V-V^{+} V V^{+}, \\
P^{(4)}=V^{+} V V^{+} V-V V^{+} V V^{+},
\end{array}
$$

which can be continued. The matrices $V$ are $4 \times 6$ matrices for 12-electron complexes and $3 \times 7$ matrices for 14-electron complexes, which organize into a single entity independent ESVs of the problem-the first order transition densities between the occupied and empty MOs of the unperturbed problem. One can check that only the even terms of the above expansion contribute to the effective charges residing on the atoms (orbital populations) of the CLS. 


\section{TCHOUGRÉEFF}

Inserting the expansion Eq. (24) rewritten in terms of matrices $V$ in the energy expression Eq. (16) with the perturbed Fockian Eq. (23) yields a DMM model of the coordination compound of an arbitrary symmetry since the transition densities $V$ take account of all possible perturbation of electronic structure keeping the CLS a separate entity. The series Eq. (24) in fact appears by expanding the closed expression for the projection operator:

$$
P=\left(P_{0}+V\right)\left(1+V^{+} V\right)^{-1}\left(P_{0}+V^{+}\right),
$$

which involves the inversion of a $10 \times 10$ matrix and nowadays is not a great computational problem. On the other hand, it is possible to restrict oneself with certain power in the expansion Eq. (24) getting to polynomial model of electronic structure of required accuracy.

It is easy to analyze the above model keeping the terms of the total order not higher than two in $\delta q$ and $V$ simultaneously and taking into account that under the spur sign the argument of the self-energy part $\Sigma$ of the Fockian can be interchanged with the matrix multiplier [25]. Using these moves we arrive to:

$$
\begin{aligned}
E_{\mathrm{CLS}}= & \underbrace{2 \mathrm{Sp}\left[h_{0} P_{0}\right]+\operatorname{Sp}\left[P_{0} \Sigma\left(P_{0}\right)\right]}_{=E_{0}}+2 \operatorname{Sp}\left[F_{0}\left(V+V^{+}\right)\right] \\
& +2 \mathrm{Sp}\left[\left(h^{\prime} \mid \delta q\right) P_{0}\right]+2 \mathrm{Sp}\left[\left(h^{\prime} \mid \delta q\right)\left(V+V^{+}\right)\right] \\
+ & \operatorname{Sp}\left[\left(V+V^{+}\right) \Sigma\left(V+V^{+}\right)\right]+\operatorname{Sp}\left[\left(\delta q\left|h^{\prime \prime}\right| \delta q\right) P_{0}\right] \\
& +2 \mathrm{Sp}\left[F_{0}\left(V V^{+}-V^{+} V\right)\right] .
\end{aligned}
$$

At the equilibrium the terms linear in $\delta q$ and $V+$ $V^{+}$vanish so that the electronic energy becomes:

$$
\begin{aligned}
E_{\mathrm{CLS}}=E_{0}+2 \operatorname{Sp}\left[\left(h^{\prime} \mid \delta q\right)\left(V+V^{+}\right)\right] \\
+\operatorname{Sp}\left[\left(V+V^{+}\right) \Sigma\left(V+V^{+}\right)\right]+\operatorname{Sp}\left[\left(\delta q\left|h^{\prime \prime}\right| \delta q\right) P_{0}\right] \\
+2 \operatorname{Sp}\left[F_{0}\left(V V^{+}-V^{+} V\right)\right],
\end{aligned}
$$

which is a quadratic form with respect to the nuclear shifts and the ESVs $V$. The average of the second derivatives of the one-electron part of the Fock operator with the operator $P_{0}$ projecting to the occupied MOs of the unperturbed system:

$$
\left(\delta q\left|2 \mathrm{Sp}\left[h^{\prime \prime} P_{0}\right]\right| \delta q\right)=\left(\delta q\left|D_{0}\right| \delta q\right)
$$

is nothing, but the bare harmonic potential of the symmetric complex with the square dynamic matrix $D_{0}$ acting on the nuclear shifts. Analogously the second order energy corrections with respect to $V$-the variation of ESVs describing one-electron density matrix:

$$
\begin{aligned}
2 \operatorname{Sp}\left[F_{0}\left(V V^{+}-V^{+} V\right)\right]+\operatorname{Sp}[(V+ & \left.\left.V^{+}\right) \Sigma\left(V+V^{+}\right)\right] \\
& =\frac{1}{2}\langle\langle V|\Lambda| V\rangle\rangle
\end{aligned}
$$

turns out to be the quadratic form giving the electronic energy as a function of the variation of the one electron density matrix. The quantity $\Lambda$ can be considered as a superoperator (supermatrix) acting in the space of the $10 \times 10$ matrices taken as elements of a linear space (the Liouville space). The supermatrix $\Lambda$ has four indices running through one-electron states in the carrier space of the CLS group. Then the formula

$$
\langle\langle A \mid B\rangle\rangle=\operatorname{Sp}\left(A^{+} B\right)
$$

defines a scalar product in the Liouville space, which ultimately allows the notation used in Eq. (27). Next move consists in forming a direct sum of the Liouville space of the of matrices $V$, which can be expanded over the basis formed by the matrix unities $|b\rangle\langle a|$ with $a$ and $b$ running over all basis states of the CLS carries space and of the space spanned by the nuclear shifts. Extending the definition of the scalar product to this new space allows to rewrite the spurs in Eqs. (25), (26) as scalar products in this vector space. Then the two types of perturbations introduced above couple by the bilinear term:

$$
2 \operatorname{Sp}\left[\left(h^{\prime} \mid \delta q\right)\left(V+V^{+}\right)\right]=\left\langle\left\langle V\left|h^{\prime}\right| \delta q\right)+\left(\delta q\left|h^{\prime}\right| V\right\rangle\right\rangle .
$$

This is nothing but the electron-vibration interaction in the chosen notation. We remind that the quantities $h^{\prime}$ are the three index supermatrices; they act, respectively, to the right on the vector of nuclear shifts producing a $10 \times 10$ matrix next forming a Liouville scalar product with matrix $V$, and on the variations $V$ of the density matrix, producing a vector to be convoluted with that of nuclear shifts $\delta q$. With use of this set of variables the energy in the vicinity of the symmetric equilibrium point becomes: 


$$
E_{\mathrm{CLS}}=E_{0}+\frac{1}{2}\left(\delta q \left\langle\left\langleV\left|\begin{array}{cc}
D_{0} & h^{\prime} \\
h^{\prime} & \Lambda
\end{array}\right| \begin{array}{l}
\delta q) \\
V\rangle\rangle
\end{array}\right.\right.\right.
$$

which is a quadratic form with respect to both the nuclear shifts and the ESVs. The substitution operator gives additional terms, which also can be recast into the form of the scalar products in the Liouville space:

$$
\begin{aligned}
h^{S}=w+w^{+} & \\
& 2 \operatorname{Sp}\left[h^{S}\left(V+V^{+}\right)\right]=\langle\langle V \mid w\rangle\rangle+\langle\langle w \mid V\rangle\rangle .
\end{aligned}
$$

With this notation the energy of the CLS becomes:

$$
\begin{aligned}
E_{\mathrm{CLS}}=E_{0}+\langle\langle V \mid w\rangle\rangle+ & \langle\langle w \mid V\rangle\rangle \\
& +\frac{1}{2}\left(\delta q\left\langle\left\langle V\left|\begin{array}{ll}
D_{0} & \left.h^{\prime} \mid \delta q\right) \\
h^{\prime} & \Lambda
\end{array}\right| V\right\rangle\right\rangle\right.
\end{aligned}
$$

This can be treated as the minimal order of the DMM picture for the PES of the coordination compounds of nontransitional elements. It perfectly condenses all the necessary elements of the LD theory of the ligand influence and of the theory of vibronic interactions. The specificity of the "class" of compounds is fixed by the presence of the CLS group. The specificity of a "subclass" within this class is controlled by the number of electrons in the CLS which defines the specific form of the quantities $P_{0}$ and $\Lambda$. Both the geometry and the electronic structure of the substituted or/and deformed complex can be obtained (in the "harmonic" approximation) by taking derivatives of the above expression with respect to $\delta q$ and $V$ and setting these former equal to zero. Doing that we see that the fixed deformation $\mid \delta q$ ) and the substitution $w$ result in the modification of the electronic structure as compared to the symmetric undeformed complex. The amount of the modification bringing the system back to the new equilibrium is given by the formula:

$$
\left.\left.\left.|V\rangle\rangle=\Lambda^{-1}\left|h^{\prime}\right| \delta q\right)+w\right\rangle\right\rangle
$$

It is remarkable that the supermatrix $\Lambda^{-1}$ is nothing (Pupyshev, Private communication) but the polarization propagator II for the CLS subsystem calculated for the symmetric molecule. With this we get:

$$
\left.\left.\left.V=\Pi\left|h^{\prime}\right| \delta q\right)+w\right\rangle\right\rangle .
$$

This performs the announced program of obtaining a closed expression for the energy of the coordination compound (or at least of its CLS) in terms of its geometry and ESVs.

\section{PES OF COORDINATION COMPOUND AS DERIVED FROM DMM}

Now we can turn to deriving a true mechanistic (MM-like) model for coordination compounds of nontransition element by excluding the ESVs $V$. Inserting Eq. (33) in Eq. (31) we get for the energy:

$$
\begin{aligned}
\frac{1}{2}((\delta q|D| \delta q) & +\left(\delta q\left\langle\left\langle h^{\prime}|\Pi| h^{\prime}\right\rangle\right\rangle \delta q\right)+\langle\langle w|\Pi| w\rangle\rangle \\
& +\left(\delta q\left\langle\left\langle h^{\prime} \mid \Pi w\right\rangle\right\rangle+\left\langle\left\langle w|\Pi| h^{\prime}\right\rangle\right\rangle \delta q\right) .
\end{aligned}
$$

This expression in a condensed form contains all the results, which are in details are obtained in [8], namely the theory of ligand influence, which can be considered as a response of molecular geometry to the chemical substitution. For example, optimizing the above expression with respect to $\mid \delta q$ ) yields the response of the complex geometry to the substitution of the ligands. One easily gets the close expression for it:

$$
\mid \delta q)=-D^{-1} \mid\left\langle\left\langle h^{\prime}|\Pi| w\right\rangle\right) .
$$

Of course, within such a formulation the effect of the substitution does not reduce to modification of the nonbonding potentials felt by the ligands (section Coulomb Contribution to Ligand Influence). By contrast the substitution affects the very substance of what is going on. Different ligands are characterized by their specific contributions to the Fockian for the CLS group. In the simplest approximation adopted in Ref. [8] the ligand is characterized by its diagonal Fockian matrix element, which is a true parameter of the model. The semiempirical SLG theory as applied to isolated ligands allows to estimate these quantities related to the LPs and even provides formulae describing their dependence on the deformations of the "organic" bonds incident to the donor atom. However, it is important to mention already now that replacing one ligand by another in a coordination compound (local perturbation) produces a nonlocal effect in that sense that it does not necessary decrease with the distance from the perturbation location (as it will be formally described later). 


\section{TCHOUGRÉEFF}

The MM-like model of complexes of nontransition elements requires even less than it is given by Eq. (34): only the first and the second term in the first row. They represent the bare harmonic dependence of the energy on the nuclear shifts and the renormalizations of the respective harmonic constants due to adjustment of the electronic structure to these shifts:

$$
D=D_{0}+\left\langle\left\langle h^{\prime}|\Pi| h^{\prime}\right\rangle\right\rangle .
$$

As we mentioned previously the specifics of the central atoms in coordination compounds is determined by the structure of the supermatrix $\Pi$, which is in its turn predefined by the structure of the carrier space of the CLS group and by the number of electrons in it. Indeed, the supermatrix $\Pi$ of the polarization propagator is particularly simple in the basis of the eigenstates of the Fock operator $F_{0}$. Its matrix elements are:

$$
\Pi_{i i^{\prime} j j^{\prime}}=\frac{\delta_{i i^{\prime}} \delta_{j j^{\prime}}}{\varepsilon_{i}-\varepsilon_{j}}
$$

where the subscripts $i i^{\prime}$ run over all occupied MOs and the subscripts $j j^{\prime}$ run over the vacant ones. In this basis the elements $V_{j i}$ of the matrix $V$ and of its conjugate by definition represent the transition densities between the $i$-th occupied and the $j$-th empty MO. They are numerical coefficients at the matrix unities $|j\rangle\langle i|$ being the basis vectors of the Liouville space. In terms of the Liouville space the superoperator $\Pi$ can be written:

$$
\Pi=\sum_{\substack{i \in \mathrm{occ} \\ j \in \operatorname{vac}}} \frac{|i \rightarrow j\rangle\rangle\langle\langle i \rightarrow j|}{\varepsilon_{i}-\varepsilon_{j}}
$$

$(|i \rightarrow j\rangle\rangle$ is the Liouville space notation for the matrix unity $|j\rangle\langle i|)$, which allows the straightforward use of the scalar product formulae with notion that:

$$
\left\langle\left\langle i \rightarrow j \mid i^{\prime} \rightarrow j^{\prime}\right\rangle\right\rangle=\left\langle i \mid i^{\prime}\right\rangle\left\langle j \mid j^{\prime}\right\rangle=\delta_{i i^{\prime}} \delta_{j j^{\prime}} .
$$

The simplest approximate description of $\Pi$ corresponds to what is known as the frontier orbitals approximation where only the highest occupied and lowest unoccupied MOs (HOMO and LUMO, respectively) are involved. Within it one gets:

$$
\Pi_{h h^{\prime} l l^{\prime}}=\delta_{h h^{\prime}} \delta_{l l^{\prime}}\left(\varepsilon_{\mathrm{H}}-\varepsilon_{\mathrm{L}}\right)^{-1}
$$

where subscripts $h h^{\prime}$ run over the orbitals in the HOMO manifold (they may be degenerate in the highly symmetric case) and $l l^{\prime}$ do the same in the possibly degenerate LUMO manifold.

The given formulae contain all necessary results but cannot be easily qualitatively interpreted. The necessary interpretation had been done by Levin and Dyachkov and is based on clarifying the interplay of the effects produced by substitution and vibronic operators upon the solution of the Hückellike problem in the 10-dimensional orbital carrier space with use of symmetry considerations. This will be done in the next section.

\section{SYMMETRY ADAPTED FORMULATION}

For the purposes of the present paper the symmetry analysis of Ref. [8] can be reformulated as follows. The deformation of the molecule of a coordination compound $\delta q$ ) is a vector with the components referring to the individual nuclear shifts:

$$
\left.\mid \delta q)=\sum_{i} \delta q_{i} \mid i\right)
$$

For a symmetric (say, octahedral) molecule it may be rewritten with use of the symmetry adapted nuclear shifts:

$$
\left.\mid \delta q)=\oplus_{\Gamma \gamma} \delta q^{\Gamma \gamma} \mid \Gamma \gamma\right)
$$

where $\Gamma$ and $\gamma$ refer, respectively, to the irreducible representation of the symmetry group and its row (in the case of a degenerate irreducible representation). In an octahedral complex if only the shifts leading to the $\mathrm{M}-\mathrm{L}(\mathrm{M}-\mathrm{X})$ bond lengths variation are concerned the symmetry classification suffice to label all possible collective shifts which can be either of $a_{1 g}, e_{g}$ or $t_{1 u}$ symmetry. They can be explicitly written through the nuclear shifts of the individual ligands according to:

$$
\begin{aligned}
\left.\left.\mid a_{1 g}\right)=\frac{1}{\sqrt{6}}\left[\mid x_{L_{x}}\right)-\mid x_{L_{-x}}\right)+\left(y_{L_{y}}\right)-\left(y_{L_{-y}}\right) & \left.+\mid z_{L_{z}}\right) \\
& \left.\left.-\mid z_{L_{-z}}\right)\right],
\end{aligned}
$$

$$
\left.\left.\left.\mid e_{g} s\right)=\frac{1}{2}\left[\left|x_{L_{x}}-\right| x_{L_{-x}}\right)-\left(y_{L_{y}}\right)+\mid y_{L_{-y}}\right)\right],
$$




$$
\begin{aligned}
\left.\left.\left.\left.\mid e_{g} c\right)=\frac{1}{2 \sqrt{3}}\left[2 \mid z_{L_{z}}\right)-2 \mid z_{L_{-z}}\right)-\mid x_{L_{x}}\right)+\mid x_{L_{-x}}\right) & -\left(y_{L_{y}}\right) \\
& \left.\left.+\mid y_{L_{-y}}\right)\right], \\
\left.\left.\mid t_{1 u} \gamma\right)=\frac{1}{\sqrt{2}}\left[\left(\gamma_{L_{\gamma}}\right)+\mid \gamma_{L_{-\gamma}}\right)\right] . &
\end{aligned}
$$

The meaning of notation for the individual nuclear shifts is that $\left.\mid \gamma_{L} \gamma\right)$ represents a unit shift in the positive direction along the $\gamma$ axis of the ligand located at the $\pm \gamma$ semiaxis of the coordinate frame.

A remarkable feature is that the derivative of one-electron part of the Fockian with respect to the symmetry adapted nuclear shift $\delta q^{\Gamma \gamma}$ (an operator acting on the one-electron states in the CLS carrier space) itself transforms according to the irreducible representation $\Gamma$ and its row $\gamma$. That means that applying the deformation $\mid \Gamma \gamma)$ to a complex results in a perturbation of the Fock operator having the same symmetry $\Gamma \gamma$. This allows to write the vibronic operator in a symmetry adapted form:

$$
\left(h^{\prime} \mid \delta_{q}\right)=\sum_{\Gamma \gamma} \delta q^{\Gamma \gamma}\left(h^{\prime}{ }_{\Gamma \gamma} \mid \Gamma \gamma\right)
$$

Finally, the substitution operator can be expanded as a sum of symmetry adapted components. For example, in the octahedral complex single substitution $\mathrm{ML}_{6} \rightarrow \mathrm{ML}_{5} \mathrm{X}$ defined in the section Coulomb Contribution to Ligand Influence results in the substitution operator:

$$
h^{S}=\frac{1}{\sqrt{6}} h_{a_{1 g}}^{S}+\frac{1}{\sqrt{3}} h_{e_{g} c}^{S}+\frac{1}{\sqrt{2}} h_{t_{1} z}^{S}
$$

As we see for the symmetric system all the elements of the present picture are classified according to irreducible representations of the relevant symmetry group $-\mathrm{O}_{h}$. For example, the energies defining the polarization propagator depend on $\Gamma_{\mathrm{H}}$ and $\Gamma_{\mathrm{L}}$ but not on the rows $\gamma_{\mathrm{H}}$ and $\gamma_{\mathrm{L}}$ of the involved irreducible representations. Using the symmetry notation for the polarization propagator allows simply realize its role as a selection mechanism for interaction of different perturbations. As we mentioned, in the frontier orbitals approximation the only energy parameter is the gap $\varepsilon_{\mathrm{H}}-\varepsilon_{\mathrm{L}}$. The polarization propagator thus acquires the form

$$
\left.\Pi=\left(\varepsilon_{\mathrm{H}}-\varepsilon_{\mathrm{L}}\right)^{-1} \sum_{\gamma_{\mathrm{H}}, \gamma_{\mathrm{L}}}\left|\gamma_{\mathrm{H}} \rightarrow \gamma_{\mathrm{L}}\right\rangle\right\rangle\left\langle\left\langle\gamma_{\mathrm{H}} \rightarrow \gamma_{\mathrm{L}}\right|\right.
$$

It is obvious that the superoperator $\Pi$ acts as a projection operator in the Liouville space cutting out those components of the $10 \times 10$ transition density matrices which mix $\gamma_{\mathrm{H}}$ state with the $\gamma_{\mathrm{L}}$ state, which is only possible if the symmetries of the perturbations both the symmetry of deformation $\Gamma_{\text {def }}$ and the symmetry of substitution $\Gamma_{\mathrm{S}}$ satisfy the selection rule:

$$
\Gamma_{\text {def }}, \Gamma_{\mathrm{S}} \subset \Gamma_{\mathrm{H}} \otimes \Gamma_{\mathrm{L}}
$$

i.e., both enter in the expansion of the tensor product of the irreducible representations of the frontier orbitals.

\section{OFF-DIAGONAL ELASTIC CONSTANTS FOR STRETCHINGS OF BONDS INCIDENT TO THE CENTRAL ATOM}

Up to this point our main concern was to reformulate the results of the LD ligand influence theory in the DMM form. Its main content was the symmetry based analysis of the possible interplay between two types of perturbation: substitution and deformation, which is controlled by the selection rules incorporated in the polarization propagator of the CLS. The mechanism of this interplay can be simply formulated as follows: substitution produces perturbations of different symmetries, which are supposed to induce transition densities of the same symmetries. In the frontier orbital approximation only those densities among all possible ones can actually appear which have the symmetry which enters into decomposition of the tensor product $\Gamma_{\mathrm{H}}$ $\otimes \Gamma_{\mathrm{L}}$ to the irreducible representations. These survived transition densities then induce the geometry deformations of the same symmetry as those former.

The deformation (nuclear shifts) may play the same role as the substitution. Inducing a deformation of some symmetry leads to appearance of the transition densities of the corresponding symmetry. The same selection rule as that for the substitution makes only the symmetry component entering into decomposition of the tensor product $\Gamma_{\mathrm{H}} \otimes \Gamma_{\mathrm{L}}$ to survive and to induce the deformation of the same symmetry. For example: the $z$-shift of the apical ligand expands as: 


$$
\left.\left.\left.\left.\mid z_{L_{z}}\right)=\left[\frac{1}{\sqrt{6}} \mid a_{1 g}\right)+\frac{1}{\sqrt{3}} \mid e_{g} c\right)+\frac{1}{\sqrt{2}} \mid t_{1 u} z\right)\right]
$$

Thus it may produce the transitional densities of the $a_{1 g}, e_{g} c$, and $t_{1 u} z$ symmetries. At this point selection rules pertinent to the frontier orbitals approximation enter: for the 12-electron complexes the symmetries of the frontier orbitals are $\Gamma_{\mathrm{H}}=e_{g}$ and $\Gamma_{\mathrm{L}}=a_{1 g}$ the tensor product $\Gamma_{\mathrm{H}} \otimes \Gamma_{\mathrm{L}}=e_{g} \otimes a_{1 g}$ $=e_{g}$ contains only the irreducible representation $e_{g}$ so that the selection rules allow only the density component of the $e_{\mathrm{g}} c$ symmetry to appear. In its turn this density induces additional deformation of the same symmetry. That means that in the frontier orbitals approximation only the elastic constant for the vibration modes of the symmetry $e_{g}$ is renormalized. This result is worth to be understood in terms of individual nuclear shifts of the ligands in the trans- and cis- positions relative to the apical one. They, respectively, are:

$$
\begin{aligned}
& \left.\left.\left.\left.\mid z_{L-z}\right)=-\left[\frac{1}{\sqrt{6}} \mid a_{1 g}\right)+\frac{1}{\sqrt{3}} \mid e_{g} c\right)-\frac{1}{\sqrt{2}} \mid t_{1 u} z\right)\right] \\
& \left.\left.\left.\mid x_{L_{x}}\right)=\left[\frac{1}{\sqrt{6}} \mid a_{1 g}\right)-\frac{1}{2 \sqrt{3}} \mid e_{g} c\right)+\frac{1}{2} \mid e_{g} s\right) \\
& \left.\left.-\frac{1}{\sqrt{2}} \mid t_{1 u} x\right)\right]
\end{aligned}
$$

Combining this all we obtain for the off-diagonal constant coupling the individual shifts of the ligands in the trans-positions to each other as:

$$
\frac{1}{3}\left(e_{g} c\left|\left\langle\left\langle h_{e_{g} c}^{\prime}|\Pi| h_{e_{g} c}^{\prime}\right\rangle\right\rangle\right| e_{g} c\right)
$$

and for the off-diagonal constant coupling the individual shifts of the ligands in the cis-positions to each other we get

$$
-\frac{1}{6}\left(e_{g} c\left|\left\langle\left\langle h_{e_{g} c}^{\prime}|\Pi| h_{e_{g} c}^{\prime}\right\rangle\right\rangle\right| e_{g} c\right)
$$

By contrast for the 14-electron complexes (nontransition nonmetals) the symmetries of the frontier orbitals are: $\Gamma_{\mathrm{H}}=a_{1 g}$ and $\Gamma_{\mathrm{L}}=t_{1 u}$ and the tensor product $\Gamma_{\mathrm{H}} \otimes \Gamma_{\mathrm{L}}=a_{1 g} \otimes t_{1 u}=t_{1 u}$ so that only the transition density corresponding to the representation $t_{1 u}$ survive. Analogous moves allow to con- clude that the off-diagonal elastic constant for stretching the trans-bonds has the form:

$$
-\frac{1}{2}\left(t_{1 u} z\left|\left\langle\left\langle h_{t_{1 u} z}^{\prime}|\Pi| h_{t_{1 u} z}^{\prime}\right\rangle\right\rangle\right| t_{1 u} z\right),
$$

whereas that for the cis-bonds vanishes.

This allows to make some predictions concerning the off-diagonal elastic constants depending on the electron count in their CLS. Due to different symmetry properties of the polarization propagator in these two cases (and according to the LD picture, which ultimately explains the qualitative difference in the stereochemistry of the 12- and 14- electron complexes), the off-diagonal constant coupling the shifts of the ligands in the trans- and cis-positions to each other in the 12-electron case is expected to have different sign. The sign of the off-diagonal coupling of the trans-positioned ligands in the 14electron case is expected to be the same as that for the cis-positioned ligands in the 12-electron case, whereas the coupling of the shifts of the cis-positioned ligands in the 14-electron case is expected to be small.

\section{MEDIUM RANGE OFF-DIAGONAL ELASTIC CONSTANTS}

In the above subsection we obtained some estimates for the off-diagonal harmonic terms coupling the stretching of different $\mathrm{M}-\mathrm{L}$ bonds incident to the central atom. The employed treatment can be extended to other types of off-diagonal terms. They originate as well from the $h^{\prime} \mathrm{II} h^{\prime}$ term in the general energy expression. The traditional $\mathrm{MM}$ picture tends to avoid the appearance such off-diagonal terms and tries to represent the energy as a sum of force fields attributed to local elements of molecular structure like bonds, etc. This implies the strictly local character of the underlying electronic structure. It is easy to understand also from a pragmatic point of view since including long-range type-specific terms in addition to those already introduced makes the entire parameterization too complicated. On the other hand, in case when the electronic structure is physically formed by not that local element as two-center bonds this must be reflected in the corresponding force fields. Incidentally, the coordination compounds possess such delocalized structure elements-the CLS-where one-electron states are extended over all atoms forming it. In 


\section{A MECHANISTIC MODEL FOR PES OF COORDINATION COMPOUNDS}

such a situation one has to expect some medium range off-diagonal harmonic couplings, i.e., specific effective coupling between the deformations occurring at the separations usually not included in the MM-like consideration. With use of the developed technique it is possible to get estimates of such "off-diagonal" elements of the harmonic molecular potential with participation of the metal atom, the very existence of which in the PES expansion is difficult to imagine if not only to stick to an informationless idea that "all must be included." As an illustrative example we consider a two-coordinated linear complex. The chemical examples are provided by the compounds of $\mathrm{Cu}^{+}, \mathrm{Ag}^{+}$, or $\mathrm{Hg}^{2+}$. In the context of the standard MM analysis it is assumed that the interactions between the atoms separated by more than three bonds are not specific and must be taken into account as nonbonded "fields" with use of the Lennard-Jones potentials. Meanwhile, using the technique presented above it can be easily shown that in the case of the above metal complexes there are specific interactions of noticeable magnitude, which according to standard scheme must be classified as the 1-5 interactions (those between the atoms separated by four bonds).

Let us consider a (metal) ion bearing as previously four vacant (one $s$ and three $p$ ) orbitals. As previously we assume that ligand molecules are represented by one LP each. In the case of linear coordination (z-axis is the molecular axis) and assuming that in the equilibrium state the LPs are directed along the bonds between the donor atoms and the metal atom the symmetry adapted combinations of the LPs have the form:

$$
\left|a_{ \pm}^{(0)}\right\rangle=\frac{1}{\sqrt{2}}(|u\rangle \pm|l\rangle) .
$$

According to [14] the LP HOs $|u\rangle$ and $|l\rangle$ (upper and lower with respect to the $z$-axis) are composed of $s$ and $p$-orbitals of the donor atom, which are directed along the unit vectors $\vec{e}_{u}$ and $\vec{e}_{l}$ :

$$
\begin{aligned}
&|u\rangle=s\left|s_{u}\right\rangle+\sqrt{1-s^{2}}\left|p_{\vec{e}_{u}}\right\rangle \\
&|l\rangle=s\left|s_{l}\right\rangle+\sqrt{1-s^{2}}\left|p_{\vec{e}_{l}}\right\rangle
\end{aligned}
$$

(with the obvious sense of $s$ as a coefficient of the corresponding s-orbital in the expansion of the corresponding $\mathrm{HO}$ ). With use of these definitions and of the symmetry considerations it is easy to identify nonvanishing matrix elements of the Fock operator acting in the CLS:

$$
\begin{aligned}
& \left\langle\sigma|h| a_{+}^{(0)}\right\rangle=\sqrt{2}\left(\beta_{\sigma \sigma}^{\mathrm{DM}} S+\beta_{\zeta \sigma}^{\mathrm{DM}} \sqrt{1-s^{2}}\right) \neq 0, \\
& \left\langle\zeta|h| a_{-}^{(0)}\right\rangle=\sqrt{2}\left(\beta_{\sigma \zeta}^{\mathrm{DM}} s+\beta_{\zeta \zeta}^{\mathrm{DM}} \sqrt{1-s^{2}}\right) \neq 0,
\end{aligned}
$$

where $\beta_{\sigma \sigma}^{\mathrm{DM}}, \beta_{\zeta \sigma}^{\mathrm{DM}}, \beta_{\sigma \zeta}^{\mathrm{DM}}$, and $\beta_{\zeta \zeta}^{\mathrm{DM}}$ are the resonance (one-electron hopping) integrals in the diatomic coordinate frame for the pairs metal-donor atom and where we denote by $\sigma$ and $\zeta$, respectively, the $s$ - and $p$-states of the metal and donor atoms, having the $\sigma$ symmetry with respect to the molecular axis (linear coordination).

The nontrivial one-electron eigenstates of the effective Fock operator for this CLS have the form:

occupied:

$$
\left|a_{+}\right\rangle=y_{+}|\sigma\rangle+x_{+}\left|a_{+}^{(0)}\right\rangle,\left|a_{-}\right\rangle=y_{-}|\zeta\rangle+x_{-}\left|a_{-}^{(0)}\right\rangle
$$

empty:

$$
\left|a_{+}^{*}\right\rangle=-x_{+}|\sigma\rangle+y_{+}\left|a_{+}^{(0)}\right\rangle,\left|a_{-}^{*}\right\rangle=-x_{-}|\zeta\rangle+y_{-}\left|a_{-}^{(0)}\right\rangle .
$$

Two more states of the $\pi$-symmetry $(|\xi\rangle$ and $|\nu\rangle)$ on the metal ion remain unchanged as in the free metal ion and both are empty. The frontier orbitals here are the $\left|a_{-}\right\rangle$(HOMO) and those in the $\pi$-manyfold $(|\xi\rangle$ and $|\nu\rangle$-LUMO).

Now let us assume that the LPs belong to polyatomic ligands. Then a valence angle MDX with a vertex at a donor atom $\mathrm{D}$ is one of the geometry variables of the molecules in the standard MM setting. We shall estimate the magnitude of the indirect (CLS mediated) interactions between variations of these valence angles. Further consideration evolves as follows. We assume the LPs to be rigidly attached to the ligands. Then changing the valence angle MDX by $\delta \chi_{u}\left(\delta \chi_{l}\right)$ yields the corresponding nonvanishing angle between the vector $\vec{e}_{u}\left(\vec{e}_{l}\right)$ and molecular axis. It respectively turns on the resonance interaction between this LP and the $|\xi\rangle$ state of the metal atom (we assume that either of the ligand LPs and the metal atom itself stay in the ( $\xi \zeta)$ plane). The corresponding matrix elements are:

$$
\begin{gathered}
\langle\xi|h| u\rangle=\beta_{\pi \pi}^{\mathrm{DM}} \sqrt{1-s^{2}} \sin \delta \chi_{u} \\
\langle\xi|h| l\rangle=\beta_{\pi \pi}^{\mathrm{DM}} \sqrt{1-s^{2}} \sin \delta \chi_{l}
\end{gathered}
$$




\section{TCHOUGRÉEFF}

where $\beta_{\pi \pi}^{\mathrm{DM}}$ is the resonance (one-electron hopping) parameter for the pair of states of the metal and donor atoms, which have $\pi$-symmetry with respect to molecular axis. The derivatives of these matrix elements (and of the Fockian itself) with respect to $\delta \chi_{u}$ and $\delta \chi_{l}$ are:

$$
\begin{array}{r}
\left.\left\langle\xi\left|\frac{\partial h}{\partial \chi_{u}}\right| u\right\rangle\right|_{\delta_{x^{\prime}}=0}=\left.\left\langle\xi\left|\frac{\partial h}{\partial \chi_{l}}\right| l\right\rangle\right|_{\delta_{x_{l}}=0}=\beta_{\pi \pi}^{\mathrm{DM}} \sqrt{1-s^{2}} \\
\left\langle\xi\left|\frac{\partial h}{\partial \chi_{l}}\right| u\right\rangle=\left\langle\xi\left|\frac{\partial h}{\partial \chi_{u}}\right| l\right\rangle=0 .
\end{array}
$$

The deformation coordinates $\left.\mid \delta \chi_{u}\right)$ and $\left.\mid \delta \chi_{l}\right)$ apparently transform according to the $\xi$-th row of the representation $\pi$ and can be further combined into the symmetric and antisymmetric adapted coordinates with respect to the plane perpendicular to the molecular axis:

$$
\begin{aligned}
&\left.\left.\left.\left.\mid \delta \chi_{+}\right)=\frac{1}{2}\left(\mid \delta \chi_{u}\right)+\mid \delta \chi_{l}\right)\right) \quad \mid \delta \chi_{-}\right)=\frac{1}{2}\left(\mid \delta \chi_{u}\right) \\
&\left.\left.-\mid \delta \chi_{l}\right)\right) .
\end{aligned}
$$

The individual deformation coordinates recover from the relations:

$$
\begin{aligned}
& \left.\left.\left.\mid \delta \chi_{u}\right)=\left(\mid \delta \chi_{+}\right)+\mid \delta \chi_{-}\right)\right) \\
& \left.\left.\left.\mid \delta \chi_{l}\right)=\left(\mid \delta \chi_{+}\right)-\mid \delta \chi_{-}\right)\right) .
\end{aligned}
$$

Assembling the relevant terms (those producing the antisymmetric $\xi$-transition densities in the CLS) we get for the off-diagonal interaction of two valence angles the following expression:

$$
K \propto \frac{\begin{array}{c}
K \delta \chi_{u} \delta \chi_{l} \\
\beta_{\pi \pi}^{2} x_{-}^{2}\left(1-s^{2}\right)
\end{array}}{4\left(\varepsilon_{p}-\varepsilon_{L}\right)}
$$

whose numerical value can be estimated as follows: for the $s p^{3}$ of the donor atoms $s^{2}=1 / 4$, the weight $x_{-}^{2}$ of the antisymmetric combination of the ligand $\mathrm{LP}$ states in the corresponding $\mathrm{HOMO}$ can be safely estimated as $2 / 3$ so that with the energy gap $\left(\varepsilon_{p}-\varepsilon_{L}\right)$ of about $5 \mathrm{eV}$ and the same value of $\beta_{\pi \pi}$ we arrive to the estimate for $K$ of $0.7 \mathrm{eV} / \mathrm{rad}^{2}$, which can be treated as if not a large but noticeable specific contribution of the 1-5 types.

\section{Discussion}

It is a widespread point of view in the MM community that the latter represents a "practical" alternative to standard quantum chemical treatments of molecular structure. On this basis the quantum mechanical models are taken as excessively complex and superfluous as compared to the problems to be solved. The problem, however, is that in the absence of such models it is difficult to estimate to what extent each specific problem possibly fits to some may be adjusted MM scheme or by contrast requires some essentially quantum mechanical approach to be solved. On the other hand just practical needs stipulate the interest in developing some MM-like models for wider classes of molecules as compared to "organic" ones for which the standard MM treatment is by many examples proven to be valid. The key point is that in fact behind any "classical" MM picture there is always covered a fairly quantum view of molecular electronic structure. As it was shown previously [17] it is possible to imagine and to successfully construct more general mechanistic models of molecular potentials (PES) than usually accepted "balls-and-springs" models of the standard MM. The derivation in Ref. [17] is based on the concept of electron group dating back to McWeeny [25] and on the "semiobservability" of these groups introduced by Ruedenberg [24]. In these terms one can state that classical MM of organic molecules implies that two-electron groups describing bonds are "semiobservable," i.e., well-defined stable groups spanning the molecular electronic structure. Then the moves described in the section Account of Deductive Molecular Mechanics result in a fairly mechanistic picture of interacting atomic tetrahedra representing the sets of orthogonal HOs, which can be further transformed to the standard MM with the externally, i.e., independently defined force field parameters. The problems faced when extending any MM-like description to other classes of molecules is the lack of understanding of the pertinent electronic group structure of the wavefunctions characteristic for the new classes of compounds to be included in the MM domain. At a first glance all the characteristics of the coordination bonds: mutual influence of the ligands, charge redistribution, dependence of magnetic properties on tiny details of molecular geometry, and composition (these latter not addressed in the present paper)—all have too much of quantum 
origin so that no mechanistic model of these properties is possible. This point of view seems to be, however, an opposite extreme. Finally the MM is quite a flexible tool, not limiting anyhow either the complexity of the force fields to be used or other characteristics of the model. In the present paper we employed the representation of the electronic structure of coordination compounds in the form of the group function product recently formalized in Ref. [17] and developed a mechanistic picture of their PES involving some necessary elements of the electronic structure description through the ESVs $\nu_{\Gamma}$ and $V$. This approach can be qualified as a DMM of the CLS group of electrons specific for the octahedral environment. For other types of coordination analogous picture can be developed, which may be useful provided the electronic structure of the molecule at hand can be described with use of the corresponding CLS group. Then using the perturbation theory the EVSs have been excluded from the consideration, thus yielding the estimates for the parameters of the force fields of more traditional form.

The models thus built remain mechanistic ones, but they naturally take into account those important features of the electronic structure, which in a standard formulation would require innumerable parameterizations for more and more tricky force fields whose form anyway remains without any fundamental basis. For example, thus obtained offdiagonal elastic constants do not assume the angular dependent form like

$$
K \sim \sin 2 \theta
$$

proposed in Ref. [6] ( $\theta$ stands for the valence angle between the bonds incident to the central ion), but suggest an existence of some more or less stable ratio between the constants describing coupling of the cis- and trans-positioned ligands. Also the obtained estimates allow to relate the sign and other characteristics of these off-diagonal constants with the chemical nature of the central atom, which is a complex problem for classical MM itself.

The performed analysis shows the weakness of all tentative attempts to include metals into "classical" MM. Within the classically looking picture possible influence effects are attributed to charge redistributions among other possibilities. In fact the charge variations are the quantities of the second order in the ESVs $V$, whereas the energy in the
DMM picture depends already on the first power of $V$. This affects the entire structure of the theory where the polarization propagator supermatrix becomes the key player defining the generalized elastic properties of molecular electronic structure expressed in terms of the ESVs $V$ in the harmonic approximation. Of course this treatment is parallel to the random phase approximation (see e.g. Ref. [25]). It is also fair to say that polarization propagators were in use when analyzing the substitution effects in the coordination compounds at a pretty early stage of these studies (see Refs. [26-28]). However, in these papers the polarization propagator was used within the reactivity indices paradigm: i.e., in order to estimate some elements of the density matrices considered as "indices of influence" rather the molecular energy itself. The general vibronic approach of Ref. [7] adopted in Ref. [8] stressed the possibility of explicit expression for the PES of substituted compounds, but did not underline the importance of the polarization propagator. This is done in the present paper.

\section{Conclusion}

In the present paper we developed a structure of possible MM of coordination compounds analyzing the electronic structure of the CLS of this class of molecules. The obtained expressions can be used either as a standalone theory of the DMM style or as a source of independent estimates of the relevant force fields in classical MM of coordination compounds.

\section{ACKNOWLEDGMENTS}

The author is thankful to Dr. I. V. Pletnev who sometime ago has drawn the author's attention to the problem of mechanistic description of coordination compounds and to Prof. A.A. Levin for valuable discussions.

\section{References}

1. Hancock, R. D. Prog Inorg Chem 1989, 37, 187.

2. Kepert, D. L. Inorganic Stereochemistry; Springer: Berlin, 1982.

3. Hay, B. P.; Hancock, R. D. Coord Chem Rev 2001, 212, 61.

4. Frenking, G.; Frölich, N. Chem Rev 2000, 100, 717. 


\section{TCHOUGRÉEFF}

5. Bersuker, I. B. Electronic Structure of Coordination Compounds; Khimiya: Moscow, 1986 (in Russian).

6. Comba, P.; Hambley, T. Molecular Modeling of Inorganic Compounds; VCH: Weinheim, 1995.

7. Bersuker, I. B. Jahn-Teller Effect and Vibronic Interactions in Modern Chemistry; Nauka: Moscow, 1987 (in Russian).

8. (a) Levin, A. A.; Dyachkov, P. N. Electronic Structure and Transformations of Heteroligand Molecules; Nauka: Moscow, 1990 (in Russian); (b) Levin, A. A.; Dyachkov, P. N. Heteroligand Molecular Systems: Bonding, Shapes and Isomer Stabilities; Taylor and Francis: New York, 2002.

9. Dewar, M. J. S.; Dougherty, R. G. The PMO Theory of Organic Chemistry. Plenum: New York, 1975.

10. Burkert, U.; Allinger, N. L. Molecular Mechanics. ACS: Washington, 1982

11. Razumov, M. G.; Melnikov, V. L.; Pletnev, I. V. J Comput Chem 2001, 22, 38.

12. Tchougréeff, A. L. J Mol Struct (THEOCHEM) 2003, 630, 243

13. Tchougréeff, A. L.; Tokmachev, A. M. Int J Quantum Chem 2004, 96, 175 .

14. Tchougréeff, A. L., Tokmachev, A. M. Int J Quantum Chem 2004, 100, 667 .
15. Tokmachev, A. M.; Tchougréeff, A. L. J Comput Chem 2005, 26, 491.

16. Tchougréeff, A. L. D.Sc. Thesis, Karpov Institute: Moscow, 2004 (in Russian).

17. Tchougréeff, A. L. J Mol Struct (THEOCHEM) 2003, 632, 91.

18. Tokmachev, A. M.; Tchougréeff, A. L. Zh Fiz Khim (Russ J Phys Chem) 1999, 73, 347.

19. Tokmachev, A. M.; Tchougréeff, A. L. J Phys Chem A 2003 , 107, 358.

20. Van Vleck, J. H. J Chem Phys 1935, 3, 803.

21. Van Vleck, J. H. J Chem Phys 1935, 3, 807.

22. Owen, J. Proc R Soc A 1955, 227, 183.

23. Tchougréeff, A. L.; Tokmachev, A. M. Int J Quantum Chem 2006, 106, 571.

24. Ruedenberg, K. Rev Mod Phys 1962, 34, 326

25. McWeeny, R. Methods of Molecular Quantum Mechanics, 2nd ed.; Academic Press: London, 1992.

26. Baranovskii, V. I.; Sizova, O. V. Teor i Eksp Khim 1974, 10, 678 (in Russian).

27. Popov, N. A. Koord Khim 1975, 1, 732 (in Russian).

28. Popov, N. A. Koord Khim 1976, 2, 1155 (in Russian). 